\title{
SOME BIOMECHANICAL ASPECTS OF THE CARPAL TUNNEL*
}

\author{
Thomas J. Armstrongt and Don B. Chaffin + \\ †Department of Environmental and Industrial Health, and $\ddagger$ Department of Industrial and Operations \\ Engineering, University of Michigan, 2260 G. G. Brown Lab., Ann Arbor, MI 48109, U.S.A.
}

\begin{abstract}
Previously presented evidence indicates that carpal tunnel syndrome is related to compression of the median nerve inside the carpal tunnel. Biomechanical arguments in which the extrinsic finger flexor tendons inside the carpal tunnel are characterized as a frictionless pulley-belt mechanism are presented to show quantitatively how wrist size, wrist position and hand position affect forces on the tendons and their adjacent structures.
\end{abstract}

\section{INTRODUCTION}

Previous investigators have suggested that carpal tunnel syndrome can be caused, precipitated or aggravated by exertions with certain hand and wrist positions (Brouwer, 1920; Brain et al., 1949; Phalen, 1966, 1972; Tanzer, 1959; Kendall, 1960; Hymovich and Lindholm, 1966; Tichauer et al., 1966, 1975, 1976; Hadler, 1977; Smith et al., 1977). It has also been reported that carpal tunnel syndrome is from two to ten times more prevalent in women than in men (Brain et al., 1947; Tanzer, 1959; Kendall, 1960; Yamaguchi et al., 1965; Czeus et al., 1966; Phillips, 1967; Phalen, 1972; Birbeck and Beer, 1975; Barranco and Strelka, 1976). There is general agreement among investigators that the incidence of carpal tunnel syndrome is related to forces on the median nerve inside the carpal tunnel. The purpose of this paper is to present biomechanical arguments that show quantitatively how forces inside flexed and extended wrists are related to wrist size, hand force and hand position.

\section{BIOMECHA NICAL ARGUMENTS}

The extrinsic finger flexor muscles, which include the flexor digitorum profundus and superficialis and the flexor pollicus longus, are major force-producing muscles during exertions of the hand. These muscles are located in the forearm and are connected to the fingers with long tendons that pass through the carpal tunnel (see Fig. 1). Deviation of the wrist from the straight position causes the extrinsic finger flexor tendons to be displaced against, and past, the adjacent walls of the carpal tunnel (Armstrong and Chaffin, 1978). When the wrist is flexed, the tendons are supported by the flexor retinaculum; when the wrist is extended, the tendons are supported by the carpal bones (see Fig. 2). According to LeVeau (1974), a tendon sliding over a curved surface is analogous to a

\footnotetext{
* Received 19 November 1978
}

belt wrapped around a pulley. The force, $F_{L}$, exerted on a pulley is a function of the belt tension, $F_{t}$, the radius of the pulley curvature, $r$, the coefficient of friction between the pulley and the belt, $\mu$, and the included angle of pulley-belt contact, $\theta$, and is expressed :

$$
F_{l},(\text { force/arc length })=\frac{F_{t} e^{\mu f}}{r} .
$$

The coefficient of tendon-trochlear friction has not been measured directly; however, friction measurements of surfaces lubricated with bovine synovial fluid indicate that the coefficient would be in the range of

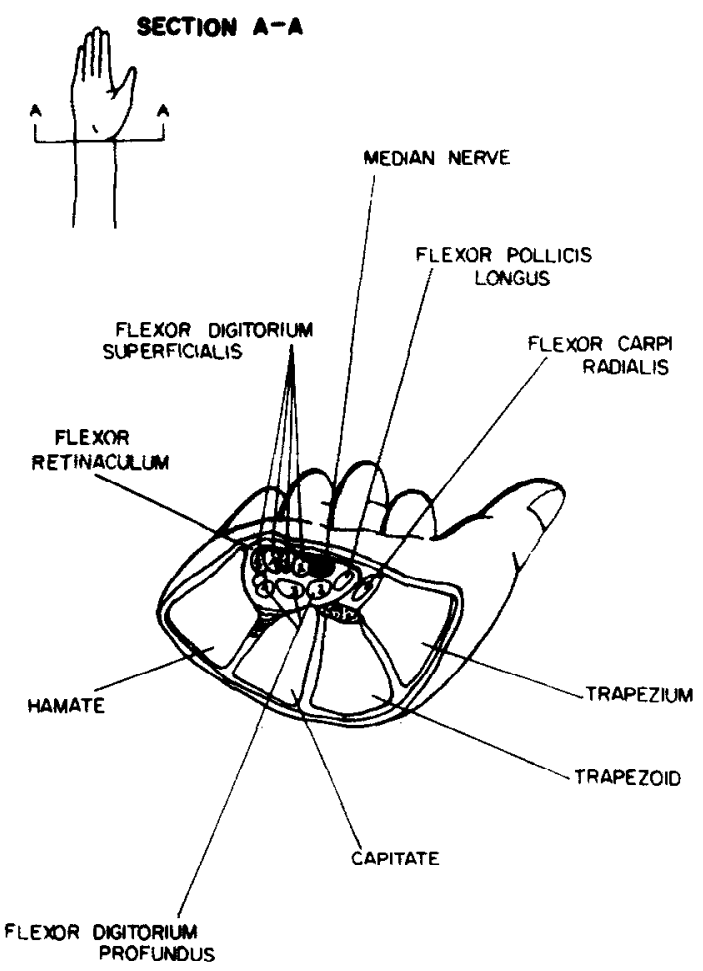

Fig. 1. A cross-sectional view of the carpal tunnel (adapted from Smith, Sonstegard and Anderson, 1977). 


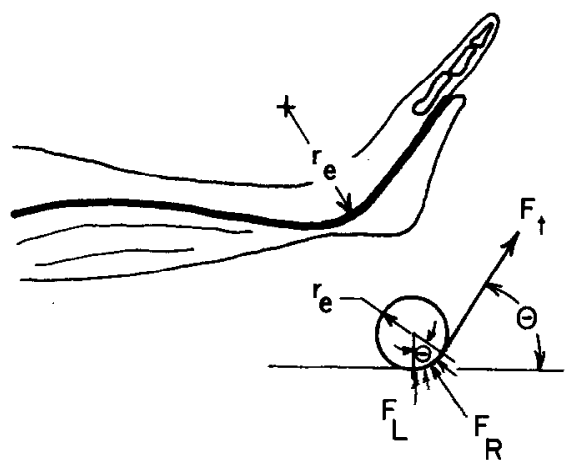

EXTENSION

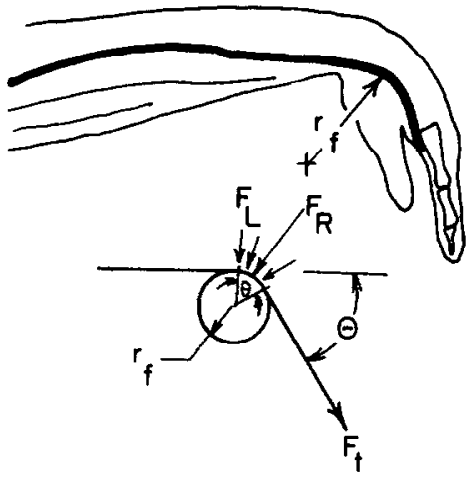

Fig. 2. During wrist extension and flexion, the extrinsic finger flexor tendons are supported by anatomical pulleys with radii $r_{e}$ and $r_{f}$, respectively. The intrawrist forces, $F_{L}$ and $F_{R}$, are described by equations (2) and (3).

0.01-0.1 (Linn, 1968; Linn and Radin, 1968). For coefficients of friction in this range, friction can be neglected without greatly affecting force estimates; thus equation (1) can be approximated by

$$
F_{L}=F_{d} / r
$$

Equation (2) indicates that the tendon load is approximately uniformly distributed over the trochlea. Tendon load per unit length as a function of tendon curvature and load is shown in Fig. 3. It can be seen that the contact force between the tendons and trochleas increases directly with tendon tension and inversely with radius of tendon curvature. The radius of curvature can be estimated for different wrist thicknesses (Armstrong and Chaffin, 1978); the tendon tension can be estimated for given positions of given sized hands (Dempster, 1961; Smith et al., 1964; Chao et al., 1976; Armstrong, 1976).

It has been suggested that force between the extrinsic finger flexor tendons and the trochlea in the flexed wrist compresses the median nerve and is a factor of carpal tunnel syndrome (Brain et al., 1949; Robbins, 1963 ; Phalen, 1966). Compression of the median nerve

* The formula for the circumference of an ellipse is

$$
c=\pi \sqrt{\frac{a^{2}+b^{2}}{2}}, \quad \text { where } \begin{aligned}
& a=\text { thickness } \\
& b=\text { breadth } \\
& c=\text { circumference. }
\end{aligned}
$$

by adjacent tendons has been confirmed by direct pressure measurements at the site of the median nerve by Tanzer (1959) and by Smith et al. (1977).

In addition to the median nerve, the synovial membranes of the radial and ulnar bursas that surround the extrinsic finger flexor tendons are compressed by forces in both flexed and extended wrists. It has been suggested that repeated compression can lead to synovial inflammation and swelling, which in turn leads to compression of the median nerve inside the carpal tunnel (Yamaguchi et al., 1965; Phalen, 1966, 1972; Tichauer, 1966, 1975, 1976).

\section{Intrawrist forces and wrist size}

Reports in the literature of wrist thicknesses for male and female populations could not be found; however, these dimensions can be estimated from wrist breadth and circumference data reported by Garrett (1970a and b) if the wrist is assumed to be shaped as an ellipse.* The thickness of a 5 percentile female wrist is thus estimated as $31.8 \mathrm{~mm}$; the thickness of a 95 percentile male wrist is estimated as $44.8 \mathrm{~mm}$. The radius of tendon curvature for the profundus tendon in extension and flexion were estimated to be 8.9 and $15.0 \mathrm{~mm}$ for the female and $\mathbf{1 2 . 0}$ and $18.1 \mathrm{~mm}$ for the male (Armstrong and Chaffin, 1978). The load distributions of the profundus tendons, based on equation (2), for a 5 percentile female and a 95 percentile male profundus tendon when the wrists are flexed and extended are shown in Fig. 3. It can be seen that when the wrist is extended, the load distribution on the 5 percentile female trochlea of the profundus tendon is $25 \%$ greater than the load on the 95 percentile male trochlea; when the wrist is flexed, the load on the female trochlea is $14 \%$ greater than the load on the male trochlea.

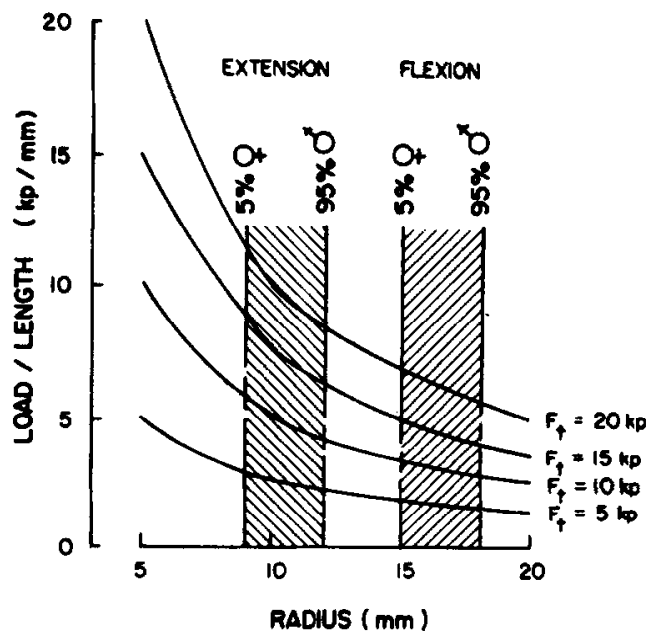

Fig. 3. Force per unit length of the flexor digitorum profundus tendon on adjacent wrist structures is shown as a function of tendon curvature and load. Tendon curvatures for 5 and 95 percentile male and female wrists were estimated from data of Garrett (1970a, b) and Armstrong and Chaffin (1978). 


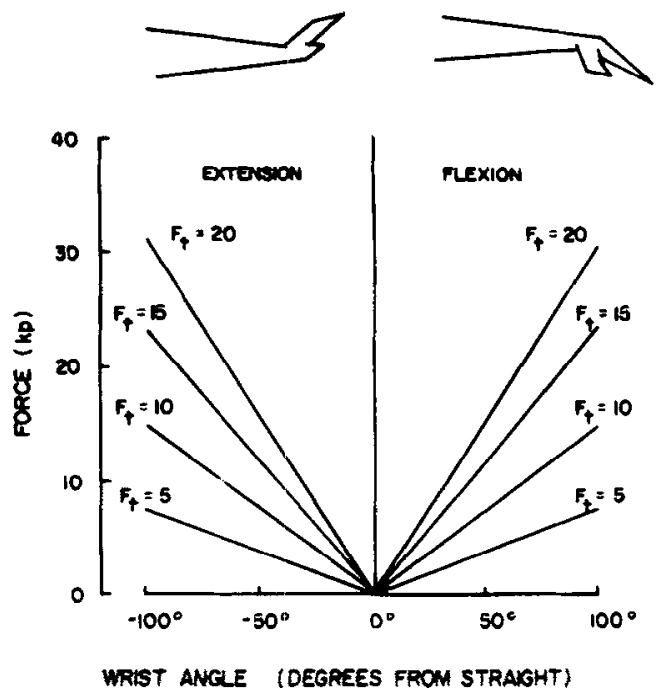

Fig. 4. The resultant force exerted by a tendon on adjacent wrist structures as a function of wrist angle and tendon load. Resultant force is independent of tendon and wrist size.

\section{Intrawrist forces and wrist positions}

The total force transmitted from a belt to a pulley depends on how far the belt is wrapped around the pulley and can be expressed as

$$
F_{R}=2 F_{\mathrm{t}} \sin (\theta / 2),
$$

where

$$
\begin{aligned}
F_{R} & =\text { resultant reaction force on tendons } \\
F_{\mathrm{z}} & =\text { tendon tension } \\
\theta & =\text { wrist angle (degrees from straight) }
\end{aligned}
$$

The resultant force is independent of the radius of tendon curvature, but it is a function of the angle of wrist deviation and of tendon load. Forces that theoretically would result at different joint angles when given tensions are exerted on a tendon are shown in Fig. 4. For a given tension, the resultant reaction force increases in an approximately linear manner with angle of deviation. Thus, exertions of the hand with a greatly deviated wrist would result in greater total force on the tendons and trochleas than would exertions with a nearly straight wrist.

\section{Intrawrist forces and hand position}

Tendon force is a function of hand size, position and load. For example, flexor digitorum profundus tendon forces were calculated from a detailed threedimensional force analysis of cadaver hands for pinch and grasp position by Chao et al. (1976). The loads of the second and third profundus tendons were calculated as 4.32 and 3.68 times the pinch force and as 2.77 and 3.05 times the grasp force. In pinch, external force is exerted only on the distal phalange, and in grasp, loads are exerted on all three phalanges. These calculations indicate that, for a given hand force, greater forces per unit length and greater resultant forces on the tendons and trochleas would be produced in pinch than in grasp.

\section{SUMMARY}

Biomechanical arguments show that the median nerve is compressed between the extrinsic finger flexor tendons and adjacent intrawrist structures during exertions of the hand with a flexed wrist. Synovial membranes surrounding the extrinsic finger flexor tendons are compressed during forceful exertions with the wrist flexed or extended. It is proposed that these biomechanical arguments support the conclusions of other investigations, that exertions with certain hand and wrist positions aggravate, precipitate or cause occupational carpal tunnel syndrome. In addition, male-female wrist size may explain in part the prevailing differences of carpal tunnel syndrome in male and female populations.

Acknowledgement - This work was supported by a grant from the National Institute for Occupational Safety and Health, No. $2001 \mathrm{OH}$ 00679-03.

\section{REFERENCES}

Armstrong, T. (1976) Circulatory and local muscle responses to static manual work. Ph.D. dissertation, University of Michigan.

Armstrong, T. and Chaffin, D. (1978) An investigation of the relationship between displacements of the finger and wrist joints and the extrinsic finger flexor tendons. $J$. Biomech. $11,119-128$.

Barranco, S. and Strelka, E. (1976) Carpal tunnel syndrome. $V$ a med. Mon. 103, 122.

Birbeck, M. and Beer, T. C. (1975) Occupation in relation to the carpal tunnel syndrome. Rheum. Rehab. 14, 218.

Brain, W., Wright, A. and Wilkson, M. (1947) Spontaneous compression of both median nerves in the carpal tunnel. Lancet 1, 277-282.

Brouwer, B. (1920) The significance of phylogenetic and ontogenetic studies for the neuropathologist. $J$. nerv. ment. Dis. 51, 113.

Chao, E., Opgrande, J. and Axmear, F. (1976) Threedimensional force analysis of finger joints in selected isometric hand functions. J. Biomech. 9, 387-386.

Cseuz, K., Thomas, J. E., Lambert, E. H., Love, J. G. and Lipscomb, P. R. (1966) Long term results of operation for carpal tunnel syndrome. Mayo clin. proc. 41, 232-241

Dempster, W. (1961) Free body diagrams as an approach to the mechanics of human posture and motion. Biomechanical Studies of the Musculo-System (Edited by Evans, F. G. et al.). Thomas, Springfield.

Garrett, J. (1970a) Anthropometry of the hands of male air force flight personnel. Aerospace Medical Research Laboratory, Aerospace Medical Division, Air Force Systems Command, Wright-Patterson Air Force Base, Ohio.

Garrett, J. (19706) Anthropometry of the air force female hand. Aerospace Medical Research Laboratory, Aerospace Medical Division, Air Force Systems Command, Wright-Patterson Air Force Base, Ohio.

Hadler, N. (1977) Industrial rheumatology: clinical investigations into the influence of the pattern of usage on the pattern of regional musculo-skeletal disease. Arthritis Rheum. 20, 1019-1025.

Hymovich, L. and Lindholm, M. (1966) Hand, wrist and forearm injuries, the result of repetitive motions. $J$. occup. Med. 8, 573-577.

Kendall, D. (1960) Aetiology, diagnosis and treatment of paraesthesia in the hands. Br. med. J. 2, 1633-1640.

LeVeau, B. (1977) Williams and Lissner: Biomechanics of Human Motion. Saunders, Philadelphia. 
Linn, F. (1968) Lubrication of animal joints. J. Biomech. 1, 193-205.

Linn, F. and Radin, E. (1968) Lubrication of animal joints. III. The effect of certain chemical alterations of the cartilage and lubricant. Arthritis Rheum. 11, 674-682.

Phalen, G. (1966) The carpal tunnel syndrome. J. Bone Jt Surg. (A) 48, 211-228.

Phalen, G. (1972) The carpal tunnel syndrome. Clin. Orthop. Rel. Res. 83, 29-40.

Phillips, R. S. (1967) Carpal tunnel syndrome as a manifestation of systemic disease. Ann. rheum. Dis. 26, 59-63.

Robbins, H. (1963) Anatomical study of the median nerve in the carpal tunnel and the etiologies of carpal-tunnel syndrome. J. Bone Jt Surg. (A) 45, 953-966.

Smith, E., Juvinall, R., Bender, L. and Pearson, J. (1964) Role of the finger flexors in rheumatoid deformities of the metacarpal-phalangeal joints. Arthritis Rheum. 7 , 457-480.

Smith, E., Sonstegard, D. and Anderson, W. (1977) Contribution of flexor tendons to the carpal tunnel syndrome. Archs phys. Med. Rehabil. 58, 379-385.

Tanzer, R. (1959) The carpal tunnel syndrome. J. Bone $J t$ Surg. (A) 41, 626-634.

Tichauer, E. R. (1966) Some aspects of stress on forearm and hand in industry. J. occup. Med. 8, 63-71.

Tichauer, E. R. (1975) Occupational biomechanics and the development of work tolerance. Paper presented at the 5th International Congress of Biomechanics, Jyvaskyla, Finland.

Tichauer, E. R. (1976) Biomechanics sustains occupational safety and health. Ind. Engng 8, 46-56.

Yamaguchi, D., Lipscomb, P. and Soule, E. (1965) Carpal tunnel syndrom. Minn. Med., 22-31. 